\title{
Real-Time Respiratory Motion Tracking: Roadmap Correction for Hepatic Artery Catheterizations
}

\author{
Selen Atasoy ${ }^{a}$, Martin Groher $^{a}$, Darko Zikic $^{a}$, Ben Glocker $^{a}$, Tobias Waggershauser $^{b}$ (MD), \\ Marcus Pfister ${ }^{c}$ and Nassir Navab ${ }^{a}$ \\ ${ }^{a}$ Computer Aided Medical Procedures (CAMP), Technische Universität München, Boltzmannstr. 3, \\ 85748 Garching, Germany, \\ \{atasoy, groher, zikic, glocker, navab\}@cs.tum.edu \\ ${ }^{b}$ Institute for Clinical Radiology, LMU - Grosshadern, Marchioninistraße 15, \\ 81337 München, Germany, \\ tobias.waggershauser@med.uni-muenchen.de \\ ${ }^{c}$ Siemens AG Medical Solutions Angio and X-Ray Systems, Siemensstr. 1, \\ 91301 Forchheim, Germany, \\ marcus.pfister@siemens.com
}

\begin{abstract}
Nowadays, hepatic artery catheterizations are performed under live 2D X-ray fluoroscopy guidance, where the visualization of blood vessels requires the injection of contrast agent. The projection of a 3D static roadmap of the complex branches of the liver artery system onto 2D fluoroscopy images can aid catheter navigation and minimize the use of contrast agent. However, the presence of a significant hepatic motion due to patient's respiration necessitates a real-time motion correction in order to align the projected vessels. The objective of our work is to introduce dynamic roadmaps into clinical workflow for hepatic artery catheterizations and allow for continuous visualization of the vessels in 2D fluoroscopy images without additional contrast injection. To this end, we propose a method for real-time estimation of the apparent displacement of the hepatic arteries in 2D flouroscopy images. Our approach approximates respiratory motion of hepatic arteries from the catheter motion in 2D fluoroscopy images. The proposed method consists of two main steps. First, a filtering is applied to 2D fluoroscopy images in order to enhance the catheter and reduce the noise level. Then, a part of the catheter is tracked in the filtered images using template matching. A dynamic template update strategy makes our method robust to deformations. The accuracy and robustness of the algorithm are demonstrated by experimental studies on 22 simulated and 4 clinical sequences containing 330 and 571 image frames, respectively.
\end{abstract}

Keywords: Abdominal Procedures, Intraoperative Imaging.

\section{DESCRIPTION OF PURPOSE}

Catheterizations of the hepatic artery are carried out during many routine treatments of liver cancer, e.g. transarterial chemoembolizations. During these minimally invasive procedures a catheter is inserted into the patient's arterial system and guided to the branches of the hepatic vessel system, which supply the cancerous tumor. Before starting the intervention a 3D roadmap (Fig. 1(a)) is acquired by 3D angiography, which provides a 3D visualization of the blood vessels with a high spatial resolution. This 3D roadmap is used to study the vasculature of the patient and to determine the blood vessels supplying the tumorous region. However, the intervention is performed under live 2D X-ray fluoroscopy guidance, which displays the catheter and parts of the patient's anatomy in real-time (12-15 frames per second) but not the blood vessels (Fig. 1(b)). For vessel visualization, multiple digital subtraction angiographies (DSA) are acquired, where each acquisition

Further author information: (Send correspondence to S.A)

S.A.: E-mail: atasoy@cs.tum.edu, Telephone: +49 (0)89 289-19427

M.G.: E-mail: groher@cs.tum.edu, Telephone: +49 (0)89 289-19427

D.Z.: E-mail: zikic@cs.tum.edu, Telephone: +49 (0)89 289-17071

B.G.: E-mail: glocker@cs.tum.edu, Telephone: +49 (0)89 289-17071

T.W.: E-mail: tobias.waggershauser@med.uni-muenchen.de, Telephone: +49 (0)89 8572663

M.P.: $\quad$ E-mail: marcus.pfister@ siemens.com, Telephone: +49 (0)9191 188951

N.N.: E-mail: navab@cs.tum.edu, Telephone: +49 (0)89 289-17057 


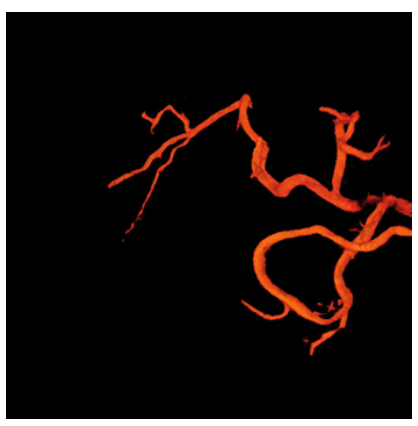

(a)

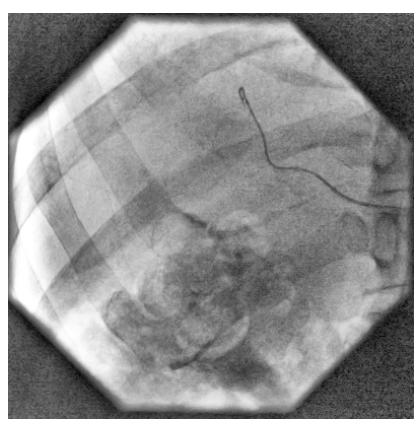

(b)

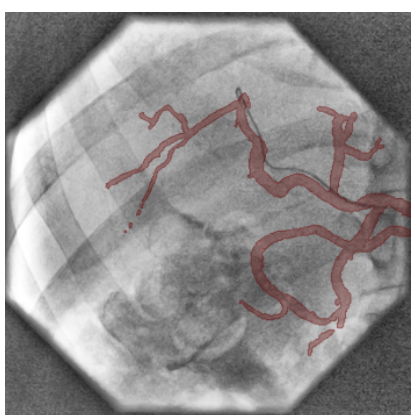

(c)

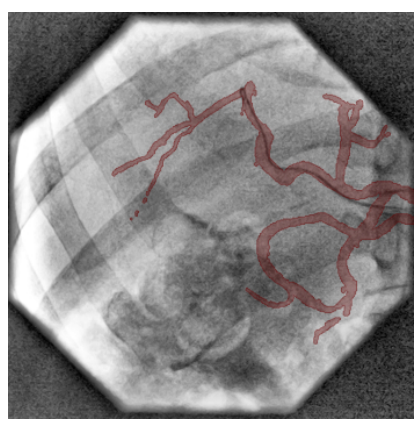

(d)

Figure 1. (a) displays the 3D static roadmap of the hepatic arteries. (b) shows a 2D fluoroscopy image. (c) illustrates the projection of $3 \mathrm{D}$ static roadmap onto the 23 . frame in the $2 \mathrm{D}$ fluoroscopy image sequence without motion correction. (d) indicates the projection of $3 \mathrm{D}$ static roadmap onto 23 . frame with motion correction.

requires the injection of contrast agent and a high X-ray radiation dose. The interventional radiologist has to mentally fuse the DSA and 2D fluoroscopy images while navigating the catheter.

Visual integration of 3D static roadmap and live 2D fluoroscopy can allow for continuous visualization of the blood vessels in fluoroscopy images without further injection of contrast agent. The projection of 3D roadmaps onto 2D fluoroscopy has been already proposed for neuroradiology. ${ }^{1,2}$ Studies for clinical evaluation of this method verify the reduction of the injected contrast agent and the procedure time. ${ }^{1}$ In liver catheterizations, however, the catheter is mostly displayed outside of the vasculature (Fig. 1(c)) due to the respiratory induced hepatic motion of the patient.

We propose a method for real-time estimation of the apparent 2D displacement of the hepatic arteries, which allows for the projection of 3D static roadmaps onto live 2D fluoroscopy in hepatic artery catheterizations. By apparent displacement we refer to the translational motion of the blood vessels projected onto the fluoroscopy image plane. However, in flouroscopy images, the vessels and thus their motion is only visible, if the vessels are contrasted. Therefore, our approach approximates the apparent displacement of the vessels by tracking the catheter motion in 2D fluoroscopy, which is the standard imaging technique for hepatic catheterizations. The computed motion is transferred to the projected 3D static roadmap and thus the projected vessels are adjusted accordingly. This way, a realistic vessel visualization in 2D fluoroscopy images is achieved with respect to the current catheter position.

\section{METHOD}

For the projection of a 3D static roadmap onto 2D fluoroscopy images, first, a rigid 2D-3D pre-registration is achieved using machine calibration. Then the apparent displacement of the vessels is computed by tracking the catheter motion. The tracking is performed on enhanced images using template matching with an appropriate motion model.

\subsection{Rigid Pre-Registration}

During the intervention, real-time 2D fluoroscopy images are acquired using a stationary vascular C-arm. State-of-theart C-arms can reconstruct a 3D roadmap (3D DSA) from two rotational runs around the patient. Acquisition of live 2D fluoroscopy images and the 3D roadmap on the same machine allows for an accurate 2D-3D registration based on machine calibration. From a previous geometric calibration, the 3D roadmap can be projected onto the live 2D fluoroscopy from any viewpoint. The feasibility of the machine-based 2D-3D registration for superimposition of 3D roadmap onto 2D fluoroscopy in neuroradiology is proved by Georges $e t a .^{2}$ In hepatic artery catheterizations, however, the produced overlay shows the projected vessels at the correct position up to a motion induced by patient's breathing.

\subsection{Motion Model}

Clifford et al. ${ }^{3}$ present an extensive assessment of respiratory hepatic motion based on nine previously published studies. All studies agree that the most significant component of liver motion is cranio-caudal translation. There exists disagreement in the literature about the significance of liver motion in anterior-posterior and lateral directions. Recent studies, 
which follow the motion of single or multiple points within the liver volume, indicate that respiration causes significant translational motion along both of these axes, whereas earlier studies, which evaluate the motion of liver margins, suggest that clinically significant liver motion can be approximated effectively by cranio-caudal translation alone. ${ }^{3}$ The tissue deformation caused by respiration is reported to be $3 \mathrm{~mm}$ on average. Rohlfing et al. ${ }^{4}$ estimate an average tissue deformation of $6 \mathrm{~mm}$ by comparison of rigid and non-rigid registration of the liver. However, they report that the rigid motion model i.e. translation and rotation accurately aligned the central area, while residual deformation occurs mostly in the periphery. ${ }^{3}$ The same group reports that the rotations did not exceed 1.5 degrees. Considering these studies we approximate the apparent displacement of the catheter due to respiration in 2D fluoroscopy images using a global translational model that covers translations in both $\mathrm{x}$ - and $\mathrm{y}$-directions in the image plane. Our method does not account for local deformations assuming that the deforming effect of respiration on the catheter is negligible.

\subsection{Approximation of Vessel Motion by Catheter Motion}

As non-contrasted hepatic arteries are not visible in 2D fluoroscopy images, our approach approximates their apparent 2D respiratory displacement from catheter motion. Since the catheter is located within the vessels, its motion inevitably comprises the vessel motion. However, the catheter motion is further complicated by deformations. Because of the branched and elastic nature of the vessels a considerable deformation of the catheter occurs while being advanced by the interventional radiologist. For the robustness of the algorithm against these catheter deformations we propose an appropriate dynamic template update strategy in Section 2.6.

\subsection{Image Enhancement}

The continuous use of X-ray fluoroscopy over a long time in hepatic catheterizations enforces the use of low radiation dose. As a consequence, the fluoroscopy images acquired during the intervention have considerably low signal-to-noise ratio (SNR) and low contrast. Thus, a successful motion tracking cannot be performed using the original images. Therefore, we apply a filtering to 2D fluoroscopy images in order to enhance the catheter and suppress noise. Enhancement of the catheter intensities also improves the influence of the tracked catheter part relative to the background during template matching and leads to more reliable estimation of the respiratory induced catheter motion.

Regarding the width of a catheter, we apply a modified Marr-Hildreth filter (Fig. 2(b)), as proposed by Wasserman et $a l .{ }^{5}$ This filter is a combination of a modified Laplace filter, which responses strong to line-like structures of width up to 5 pixels such as catheter, and a Gaussian filter, which leads to suppression of noise in the images. Because of its symmetric form the filter can be implemented in an efficient way by considering overlapping parts. The complexity of the filtering step can be reduced from $O\left(n^{2} N^{2}\right)$ to $O\left(n N^{2}\right)$, where $n$ denotes the width (or height) of the filter and $N$ denotes the width (or height) of the image. Thus, the filtering step can be accelerated approximately 10 times, which is an important property for the real-time constraint.

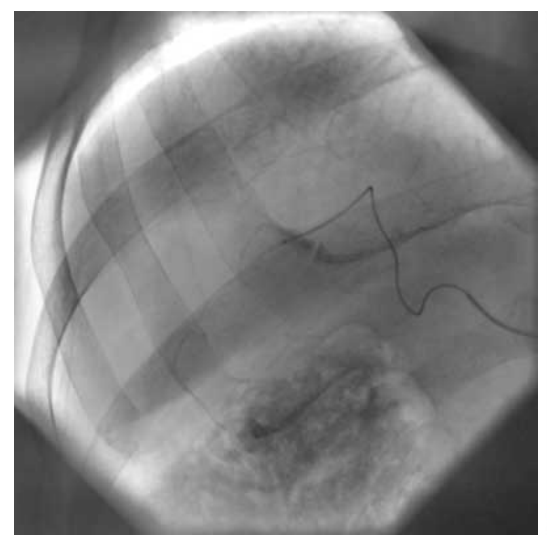

(a)

\begin{tabular}{|ccccccccccc|}
\hline 3 & 3 & 3 & 3 & 3 & 3 & 3 & 3 & 3 & 3 & 3 \\
3 & 7 & 7 & 7 & 7 & 7 & 7 & 7 & 7 & 7 & 3 \\
3 & 7 & 1 & 1 & 1 & 1 & 1 & 1 & 1 & 7 & 3 \\
3 & 7 & 1 & -3 & -3 & -3 & -3 & -3 & 1 & 7 & 3 \\
3 & 7 & 1 & -3 & -20 & -20 & -20 & -3 & 1 & 7 & 3 \\
3 & 7 & 1 & -3 & -20 & -148 & -20 & -3 & 1 & 7 & 3 \\
3 & 7 & 1 & -3 & -20 & -20 & -20 & -3 & 1 & 7 & 3 \\
3 & 7 & 1 & -3 & -3 & -3 & -3 & -3 & 1 & 7 & 3 \\
3 & 7 & 1 & 1 & 1 & 1 & 1 & 1 & 1 & 7 & 3 \\
3 & 7 & 7 & 7 & 7 & 7 & 7 & 7 & 7 & 7 & 3 \\
3 & 3 & 3 & 3 & 3 & 3 & 3 & 3 & 3 & 3 & 3 \\
\hline
\end{tabular}

(b)

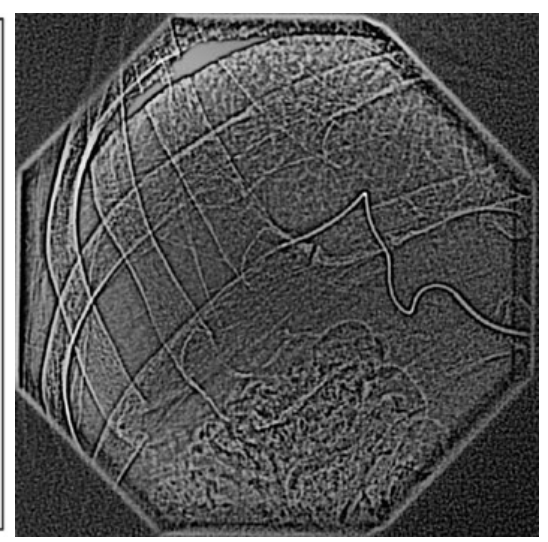

(c)

Figure 2. (a) shows a 2D fluoroscopy image. (b) illustrates the modified Marr-Hildrecht filter. (c) displays the filtered image. 


\subsection{Motion Tracking}

After the filtering step the apparent 2D displacement of the catheter is estimated using template matching. Thereby, the displacement $\mathbf{d}=\left(d_{x}, d_{y}\right)$ of a structure between two frames is computed by defining a template containing this structure in one frame and by finding the matching region in the other frame. The matching is defined in respect to a chosen similarity measure. Because of its robustness against noise and linear illumination changes we use the Correlation Coefficient (CC) as a similarity measure. Using $\mathrm{CC}$ the similarity between a template $T$ and a region of image $I$ can be determined as:

$$
C C(T, I, \mathbf{d})=\frac{\sum_{\mathbf{x} \in T}\left(T(\mathbf{x})-\mu_{T}\right)\left(I(\mathbf{x}+\mathbf{d})-\mu_{I, T}\right)}{\sqrt{\sum_{\mathbf{x} \in T}\left(T(\mathbf{x})-\mu_{T}\right)^{2} \sum_{\mathbf{x} \in T}\left(I(\mathbf{x}+\mathbf{d})-\mu_{I, T}\right)^{2}}}
$$

where

$$
\mu_{T}=\frac{1}{W H} \sum_{\mathbf{x} \in T} T(\mathbf{x}) \quad \text { and } \quad \mu_{I, T}=\frac{1}{W H} \sum_{\mathbf{x} \in T} I(\mathbf{x}+\mathbf{d})
$$

are the mean values of the intensities within the template and within the respective window in the image. Considering the definition of covariance and standard deviation Equation 1 can be rewritten as:

$$
C C(T, I, \mathbf{d})=\frac{\sum_{\mathbf{x} \in T} T(\mathbf{x}) I(\mathbf{x}+\mathbf{d})-\sum_{\mathbf{x} \in T} T(\mathbf{x}) \sum_{\mathbf{x} \in T} I(\mathbf{x}+\mathbf{d})}{\sqrt{\left(\sum_{\mathbf{x} \in T} T(\mathbf{x})^{2}-\frac{1}{W H}\left(\sum_{\mathbf{x} \in T} T(\mathbf{x})\right)^{2}\right)\left(\sum_{\mathbf{x} \in T} I(\mathbf{x}+\mathbf{d})^{2}-\frac{1}{W H}\left(\sum_{\mathbf{x} \in T} I(\mathbf{x}+\mathbf{d})\right)^{2}\right)}},
$$

which allows for canceling the mean computation for each pixel position. Thus, Equation 3 is particularly well suited for the efficient implementation of CC concerning our real-time constraint. The displacement $\mathbf{d}=\left(d_{x}, d_{y}\right)$ of the structure between the two frames is then defined as:

$$
\mathbf{d}^{\star}=\arg \max _{\mathbf{d}} C C(T, I, \mathbf{d})
$$

For estimating the global translational motion we only consider a small part of the catheter. The part has to contain both a vertical and a horizontal component in order to avoid the well-known aperture problem. In the initialization step of the tracking algorithm we ask the interventional radiologist to draw a small rectangle in the first frame covering such a part of the catheter. This region is then used as template. Template matching is performed in a search region bigger than the largest expected translation induced by respiratory motion (up to $9 \mathrm{~mm}$ in $\mathrm{x}$ - and $45 \mathrm{~mm}$ in y-direction, refer to Clifford et $a l .^{3}$ ) (Fig. 3). For tracking the motion between consecutive frames the search region is translated with the last computed displacement for each frame and the template matching is invoked in a smaller search region (template width plus $4 \mathrm{~mm}$ in $\mathrm{x}$ - and template height plus $17 \mathrm{~mm}$ in $\mathrm{y}$ - proved to be sufficient in our experiments). With this technique, the search region is kept small, which provides an acceleration in runtime of the algorithm and also decreases the probability of matching the catheter with other line-like structures such as bones. Moreover, the large influence of the catheter within the small template assures a reliable response of the $\mathrm{CC}$ for estimating the catheter motion induced by breathing.

\subsection{Dynamic Template Update}

Using the same template for the entire intervention involves the assumption that the appearance of the catheter part contained in the template remains the same over time. As the appearance of the catheter changes due to its deformation induced by the radiologist's movement, the algorithm will fail in general using only the template created from the first frame. A naive solution is to update the template every frame (or every $\mathrm{n}$ frames). The problem with this approach is that each time the template is updated small errors are introduced due to drift of the template. Matthews et al. ${ }^{6}$ propose a method with drift correction, where the template update is only performed if the new template leads to results close to the one given by the original template. The underlying assumption behind this strategy is that there must be a problem with the new template if the difference between the two results is large. However, in our case the catheter deformation necessitates the template update. Therefore, unlike Matthews et al. we update the template only if there is a considerable change in the structure contained in the template assuming that the reason of the change is the catheter deformation. This is considered if the best $\mathrm{CC}$ value of the current frame is lower than a threshold. 


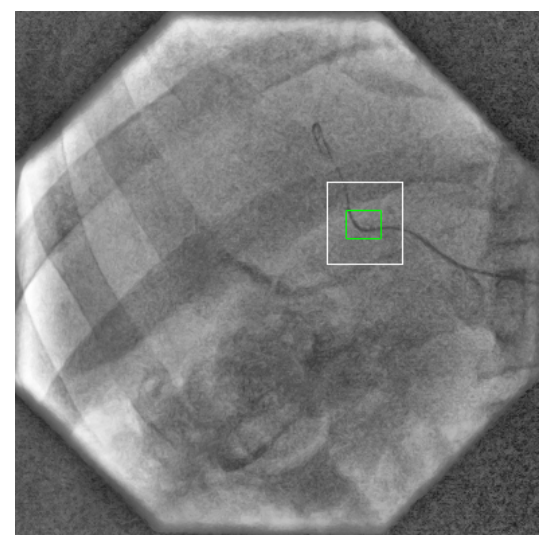

(a)

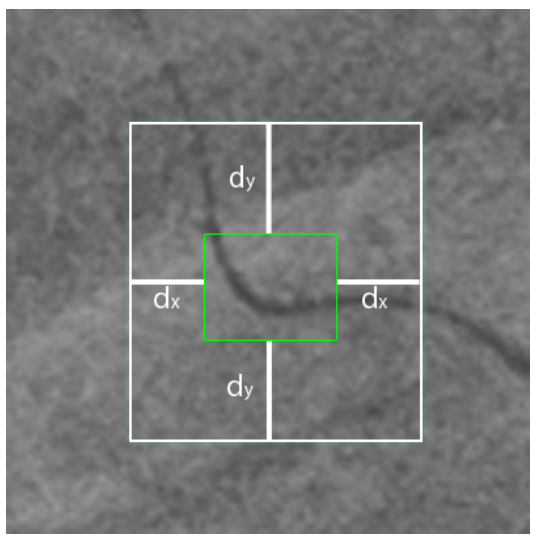

(b)

Figure 3. This figure illustrates an example for the template and the size and position of the restricted search region for templatematching, where the green rectangle denotes the template and the larger white rectangle shows the search region with respect to selected template. $d_{x}$ and $d_{y}$ illustrate the maximum expected displacement in $\mathrm{x}$ - and y-direction respectively.

Since the values of the CC depend on the created template, we determine a template specific threshold $t$ as $t=C C_{\max }-\varepsilon$. $C C_{\max }$ is the maximum CC value between the template (created from the first frame) and the second fluoroscopic image frame. $\varepsilon$ is a tolerance value, which is determined empirically in our experiments. A value of 0.35 yields the best results in all our data sets. If the template update is performed, we use the region that yields the maximum score of CC in the previous frame as the new template.

\section{RESULTS}

The accuracy of the algorithm and its robustness against noise and catheter deformation is tested using both simulated and clinical image sequences.

\subsection{Simulation Studies}

In order to perform a quantitative analysis with a known ground truth, we generate image sequences by artificially superimposing a catheter onto real 2D fluoroscopy sequences of 15 frames.

\subsubsection{Creation of Simulated Images}

In the simulated images, the catheter is manually removed and the corresponding region is inpainted by bilinear interpolation (Fig. 4(b)). In order to create a realistic catheter in terms of image intensity characteristics of 2D X-ray fluoroscopy images, the catheter intensities are computed according to actual catheter intensities in the image sequence. Therefore, the mean and standard deviation of the catheter intensities in the original sequence are computed and the artificial catheter is added to the simulated background sequence while selecting its intensities randomly with respect to the computed mean and standard deviation. By using clinical images and real catheter intensities, we assure that the simulated images are realistic (Fig. 4(c)).

The position of the catheter is changed over the frames by a translation in $y$ direction, which is to be corrected with our method. Starting with zero displacement, over the first 7 frames, the total displacement is increased by $4.8 \mathrm{~mm}$ between single frames, and in the second 7 frames, it is decreased by the same amount, resulting in a rough approximation of the respiratory motion visible in the images. This results in a maximal displacement of $33.6 \mathrm{~mm}$. Using this setting, we perform two tests: one in which the added catheter is deformed, and a second, in which the robustness to noise is tested. 


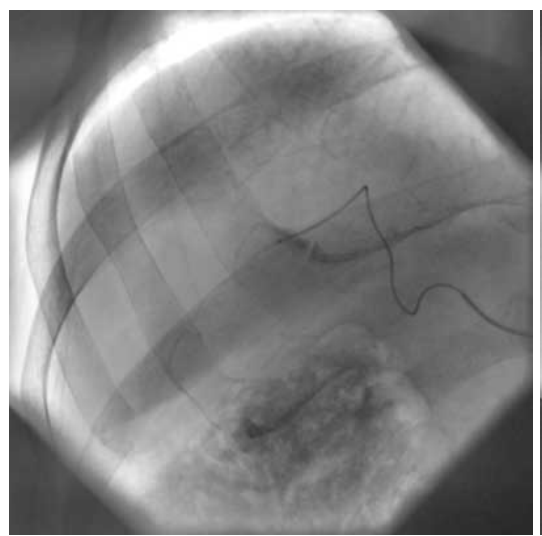

(a)

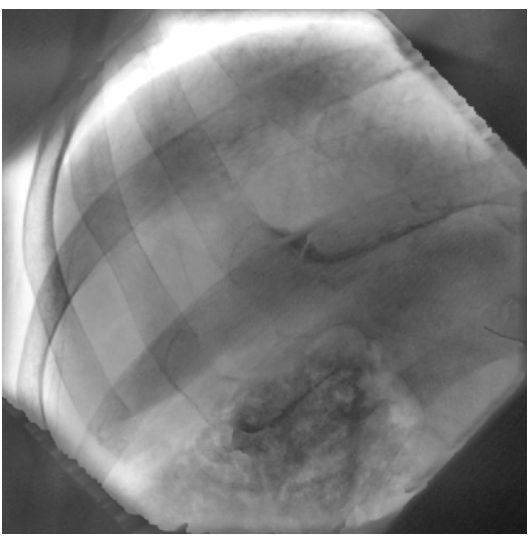

(b)

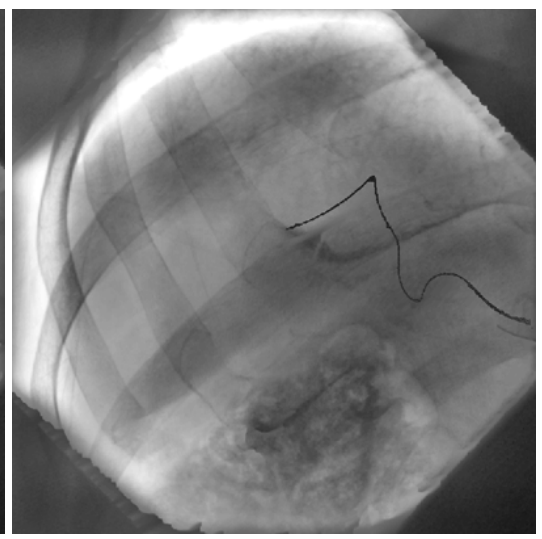

(c)

Figure 4. (a) shows a 2D fluoroscopy image. (b) illustrates the background image created by removing the catheter and inpainting the corresponding region. (c) shows the simulated image with the artificial catheter.

\subsubsection{Catheter Deformation}

The accuracy of the algorithm in presence of catheter deformation is tested on the described sequence in 11 tests with different magnitudes of deformation (10-60 pixels corresponding to 2.4-14.6 mm), resulting in a total of 165 simulated frames. To this end, the artificial catheter is deformed, before adding it to the background sequence (Fig. 5(b)). The deformation is performed moving 100 points of a free-form deformation (FFD) model based on cubic B-splines with a random displacement between 0 and a threshold. Naturally, the degree of the resulting deformation increases with the threshold for random motion of control points (Fig. 5(a)-5(b)). In Table 1 the success rate of our algorithm is shown with respect to different degrees of deformation, where $d_{\max }$ denotes the used threshold. For all magnitudes of catheter deformation, the mean error of the computed displacements remains small $(0.30-1.21 \mathrm{~mm})$.

\subsubsection{Image Noise}

The influence of noise is tested by adding different amounts of Gaussian noise to the sequence presented above in 11 tests, resulting in 165 simulated image frames. In this test, no deformation is added to the catheter. Note that the simulated images also contain the regular amount of signal dependent Poisson noise present in the X-ray beams, since they are created by manipulating clinical image sequences. By an additional Gaussian noise with variance of between $0.5 \%$ and $5.5 \%$ of the maximum image intensity (Fig. 5(c)) the mean error of the algorithm is between $0.00 \mathrm{~mm}$ and $0.85 \mathrm{~mm}$. Table 2 presents the results of our algorithm applied on simulated image sequences with additional noise, where Var. denotes the variance and accordingly the amount of the Gaussian noise. In conclusion, based on these result we can report that the algorithm is robust against noise up to high level, which is normally not encountered in 2D X-ray fluoroscopy images.

\subsection{Patient Data Studies}

In order to evaluate the accuracy of the algorithm on real data we manually segment the catheter in 164 frames of a clinical image sequence and measure the fraction of catheter contained in the vessel by computing $\left|N_{C} \cap N_{R}\right| /\left|N_{C}\right|$, with $N_{C}$ and $N_{R}$ being the segmented pixels of the catheter and the roadmap respectively. Our motion correction improves the catheter overlap from $36 \%$ to $70 \%$. This improvement is very promising considering the fact that a correction of the total apparent motion using only the non-contrasted 2D fluoroscopy images is an ill-posed problem due to the complex motion of blood vessels with an advancing catheter inside.

The influence of template size and location on the success of the algorithm is studied on 4 clinical fluoroscopy image sequences (with a total of 571 frames). For that, three inexperienced users select three points on the catheter for each sequence. Centered at each point 38 templates with different sizes between $3 \times 3$ pixels and $150 \times 150$ pixels are created. During the tests the size of the search region is set to the size of the template plus an extension in $x$ and $y$ direction of 20 and 40 pixels, respectively. The algorithm is then performed using each template. In the absence of an absolute ground truth, we compare the results to the displacements determined by manually defined motion correction. For each user the 


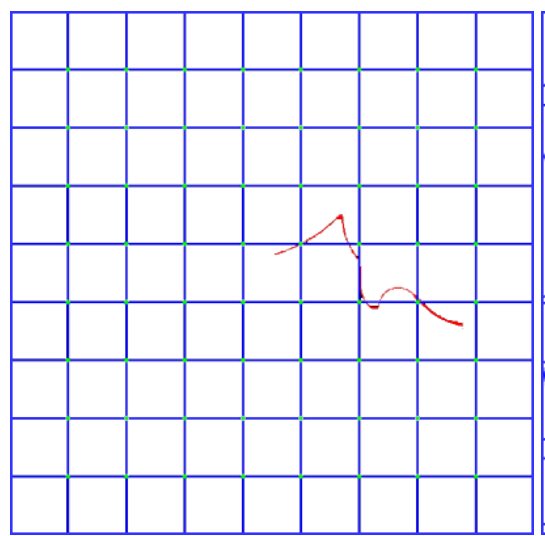

(a)

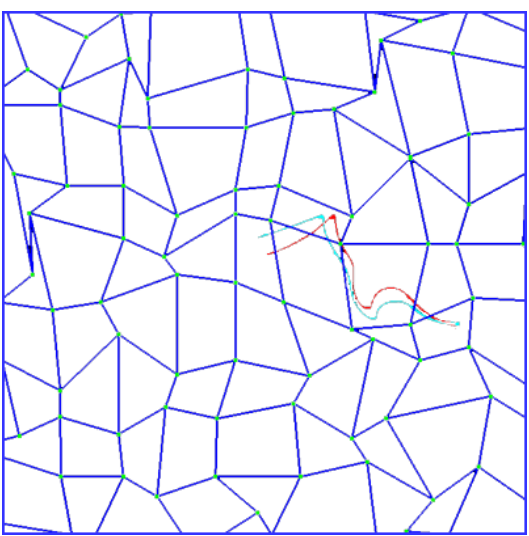

(b)

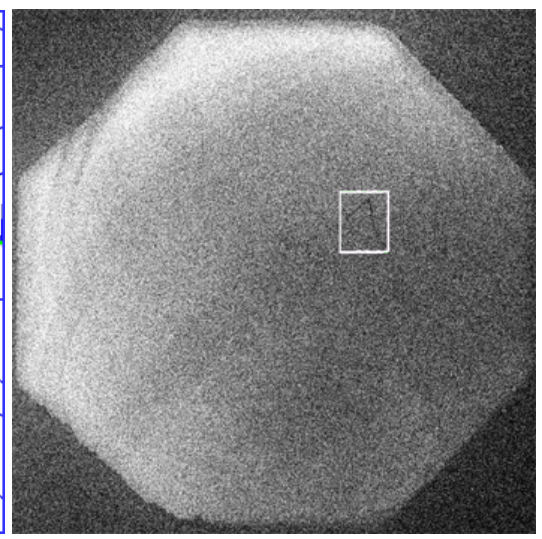

(c)

Figure 5. (a) shows the grid with 100 control points used to deform the artificial catheter. (b) illustrates the catheter deformation for a threshold of 60 pixels $(14.6 \mathrm{~mm})$, where the catheter before deformation is shown in red and the one after deformation is shown in cyan. (c) displays the effect of additional Gaussian noise with a variance of 5.5\% of the image intensities, where the rectangular region is used as template by motion tracking.

\begin{tabular}{|cccc|}
\hline $\begin{array}{c}d_{\text {max }} \\
{[\mathrm{px}]}\end{array}$ & $\begin{array}{c}\text { Error }_{x} \\
{[\mathrm{px}]}\end{array}$ & $\begin{array}{c}\text { Error }_{y} \\
{[\mathrm{px}]}\end{array}$ & $\begin{array}{c}\text { Error }_{\mu} \\
{[\mathrm{mm}]}\end{array}$ \\
\hline \hline 10 & 4 & 2 & 0.304 \\
\hline 15 & 4 & 5 & 0.514 \\
\hline 20 & 4 & 4 & 0.359 \\
\hline 25 & 5 & 3 & 0.402 \\
\hline 30 & 4 & 20 & 1.033 \\
\hline 35 & 3 & 7 & 0.665 \\
\hline 40 & 3 & 7 & 0.665 \\
\hline 45 & 5 & 7 & 0.935 \\
\hline 50 & 5 & 10 & 1.016 \\
\hline 55 & 5 & 13 & 1.089 \\
\hline 60 & 5 & 16 & 1.210 \\
\hline
\end{tabular}

Table 1.

\begin{tabular}{|cccc|}
\hline Var. & $\begin{array}{c}\text { Error }_{x} \\
{[\mathrm{px}]}\end{array}$ & $\begin{array}{c}\text { Error }_{y} \\
{[\mathrm{px}]}\end{array}$ & $\begin{array}{c}\text { Error }_{\mu} \\
{[\mathrm{mm}]}\end{array}$ \\
\hline \hline 0.005 & 0 & 0 & 0 \\
\hline 0.010 & 0 & 0 & 0 \\
\hline 0.015 & 0 & 0 & 0 \\
\hline 0.020 & 1 & 1 & 0.023 \\
\hline 0.025 & 1 & 1 & 0.077 \\
\hline 0.030 & 1 & 2 & 0.096 \\
\hline 0.035 & 1 & 2 & 0.151 \\
\hline 0.040 & 1 & 3 & 0.157 \\
\hline 0.045 & 2 & 2 & 0.248 \\
\hline 0.050 & 2 & 15 & 0.491 \\
\hline 0.055 & 3 & 15 & 0.897 \\
\hline
\end{tabular}

Table 2.

In both tables Error $x$ and Error $y$ denote maximum difference between the expected and computed displacement in $x$ and $y$ directions in pixels and Error ${ }_{\mu}$ denotes average error over all frames in mm. $d_{\max }$ in Table 1 denotes the threshold for the random displacements of the control points. Var. in Table 2 denotes the variance of the additional Gaussian noise.

average and standard deviation of the mean error over all frames is displayed in Fig. 6(a) and 6(b) respectively. The error in one frame is computed as $\left\|\mathbf{d}_{\mathbf{e}}-\left(\mathbf{d}_{\mathbf{p}_{1}}+\mathbf{d}_{\mathbf{p}_{2}}+\mathbf{d}_{\mathbf{p}_{3}}\right) / 3\right\|$, where $\mathbf{d}_{\mathbf{e}}$ denotes the expected displacement and $\mathbf{d}_{\mathbf{p}_{1}}, \mathbf{d}_{\mathbf{p}_{2}}, \mathbf{d}_{\mathbf{p}_{3}}$ denote the computed displacements using template centered at points $p_{1}, p_{2}$ and $p_{3}$, respectively. For all templates of size between $20 \times 20$ and $80 \times 80$ the error of the algorithm is in average $1.25 \mathrm{~mm}$ with a standard deviation less than 0.44 $\mathrm{mm}$. Such an error is visually barely noticeable and admissible for hepatic artery catheterizations. This study shows that the method still leads to near-optimal results for a large range of template sizes and for different template locations. Thus, we can conclude that the method does not require a precise template creation but involves just a simple user interaction, which is admissible for hepatic artery catheterizations as confirmed by our clinical partners. 


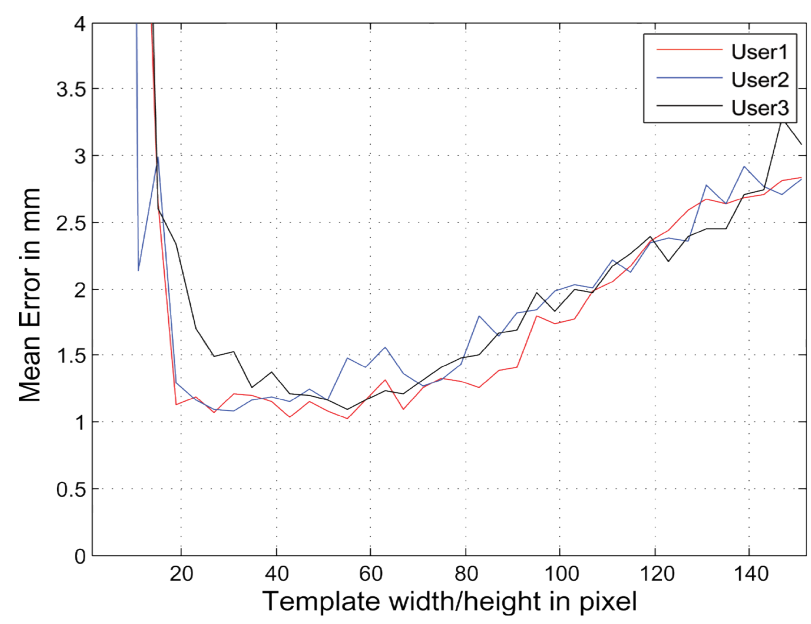

(a)

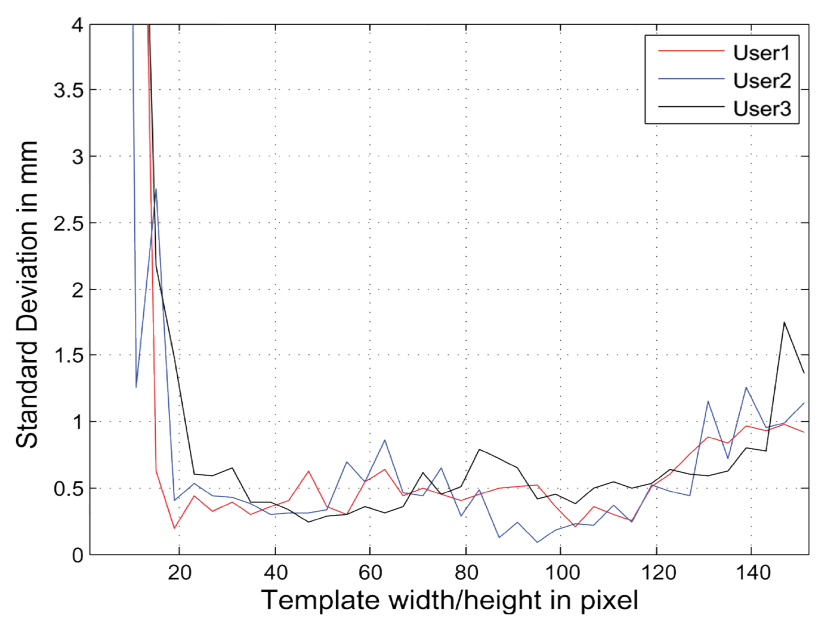

(b)

Figure 6. (a) and (b) illustrate the average and standard deviation of the error in patient data study with respect to template size.

\section{NEW OR BREAKTHROUGH WORK TO BE PRESENTED}

We consider the following points to be the major contribution of the presented work. The overlay of $3 D$ roadmaps onto $2 D$ flouroscopy sequences of the abdomen has not been previously presented in the literature, most probably due to the lack of motion correction. To the best of our knowledge, this is the first method for estimation of apparent respiratory motion based only on monoplane fluoroscopy images, which performs in real-time and results in an improved roadmap overlay onto live fluoroscopy images.

\section{CONCLUSION}

In this paper, we present an image based method for correction of apparent displacement on projections of 3D static roadmaps. Our method allows for the overlay of 3D static roadmap onto live 2D fluoroscopy in hepatic artery catheterizations despite the respiratory motion. The objective of our work is to introduce the dynamic roadmaps into the clinical routine of in hepatic artery catheterizations in order to aid interventional radiologists in catheter navigation (See video 7(a)). Our clinical partners consider the use of dynamic roadmaps during the intervention very beneficial, since it can minimize the use of contrast agent and reduce the X-ray radiation dose and the intervention time. The reduction of the injected contrast agent and intervention time when projecting the 3D roadmap onto live 2D fluoroscopy has already been demonstrated for neuroradiology. ${ }^{1}$ We plan to evaluate our approach for the projection of the 3D roadmap onto live 2D fluoroscopy in hepatic catheterizations.

\section{REFERENCES}

1. M. Söderman, D. Babic, R. Homan, and T. Andersson, "3D roadmap in neuroangiography: technique and clinical interest," Neuroradiology 47(10), pp. 735-740, 2005.

2. S. Gorges, E. Kerrien, M. Berger, Y. Trousset, J. Pescatore, R. Anxionnat, L. Picard, and S. Bracard, "3D Augmented Fluoroscopy in Interventional Neuroradiology: Precision Assessment and First Evaluation on Clinical Cases," Workshop on Augmented environments for Medical Imaging and Computer-aided Surgery - AMI-ARCS 2006 , 2006.

3. M. Clifford, F. Banovac, E. Levy, and K. Cleary, "Assessment of hepatic motion secondary to respiration for computer assisted interventions,” Computer Aided Surgery 7(5), pp. 291-299, 2002.

4. T. Rohlfing, C. Maurer Jr, W. ODell, and J. Zhong, "Modeling liver motion and deformation during the respiratory cycle using intensity-based nonrigid registration of gated MR images," Medical Physics 31, p. 427, 2004.

5. D. Palti-Wasserman, A. Brukstein, and R. Beyar, "Identifying and tracking a guide wire in the coronary arteries during angioplasty from x-ray images," IEEE Trans. on Biomedical Engineering. (TBE) 44(2), pp. 152-164, 1997.

6. L. Matthews, T. Ishikawa, and S. Baker, "The Template Update Problem," IEEE Trans. Pattern Analysis and Machine Intelligence (PAMI) 26(6), pp. 810-815, 2004. 


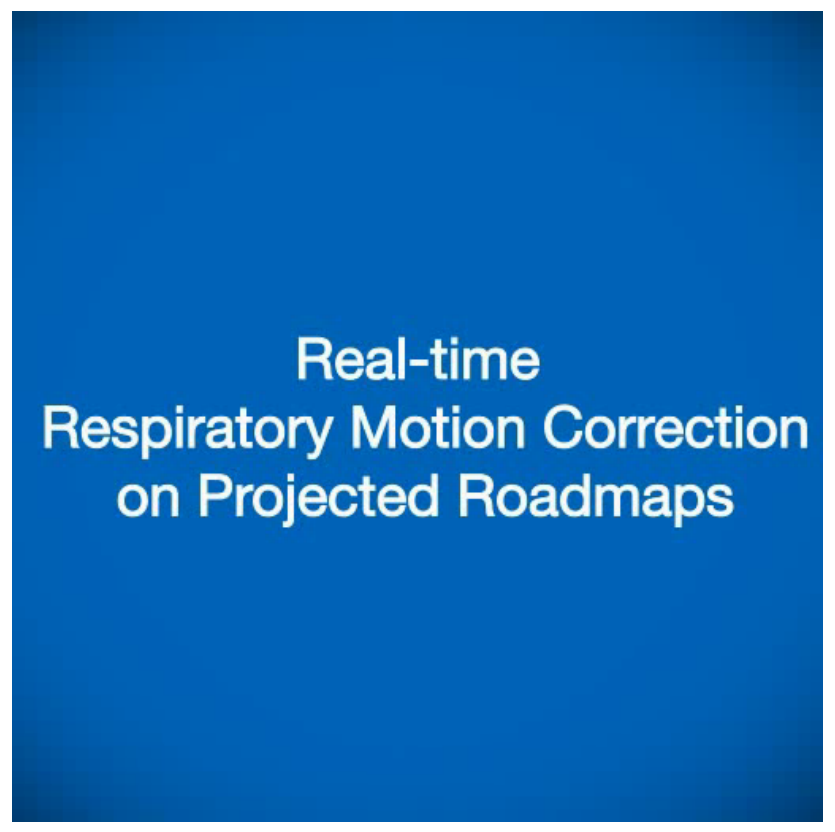

(a)

Figure 7. This video illustrates the results of our proposed motion correction algorithm by the projection of a 3D roadmap onto live 2D fluoroscopy. 